\title{
The Design of X-Ray Film Reader with Film Presence Detector
}

\author{
Tatiya Padang Tunggal ${ }^{1}$, Muhtadin Arrosyid ${ }^{2}$, Gabriel De Brito Silva ${ }^{3}$, Andino Maseleno ${ }^{4}$, Omar tanane ${ }^{5}$ \\ ${ }^{1,2}$ Department of Electrical Engineering, Universitas Muhammadiyah Yogyakarta, Yogyakarta, Indonesia \\ ${ }^{3}$ School of Engineering and Applied Sciences, Nile University, Giza, Egypt \\ ${ }^{4}$ Institute of Informatics and Computing Energy, Universiti Tenaga Nasional, Malaysia. \\ ${ }^{5}$ Faculty of Sciences Ben M'sik, University Hassan II of Casablanca, Casablanca, Morocco \\ tatiyapt@umy.ac.id, MuhtadinArrosyid@umy.ac.id, gabriel.silva@eu4m.eu, andimaseleno@gmail.com, \\ omar.tanane@univh2c.ma
}

\begin{abstract}
X-Ray viewer is a tool for observing the results of $X$-Ray films using ray lighting. It aims to get clearer readings of $\mathrm{X}$-Ray films by radiographers and doctors. X-Ray viewers in hospitals generally cannot be carried anywhere because they use fluorescent lamps as a source of radiation and use 220 Volt AC voltage directly. So that its use is less effective and efficient because it must be connected directly to a 220 Volt AC power source and requires large power. In this regard the author wants to design an X-Ray viewer tool that can be used to read the results of $X$-Ray films clearly and is portable so that the device can be used anywhere because it uses a battery as a voltage source and is equipped with a presence detection sensor film in order to save energy so that the use of tools is more effective and efficient.
\end{abstract}

Keywords - tachometer, rotation per minute, microcontoller

\section{INTRODUCTION}

$\mathrm{X}$-Ray film is a supporting medical device in the hospital that plays an important role in the analysis of disorders of the bones and other body organs. X-Ray film is a medium that is used to record the shadow of an object in the form of an organ that is recorded by X-ray irradiation. The result of the film is used by the user to analyze parts of the body that have been irradiated.

So far, there are no x-ray film reader yet which can be used portable and equipped with a film presence sensor, so a reader is made portable X-Ray film with a built-in sensor its use is more effective. In reading the results of X-Ray films by the user, required a lighting tool that is evenly (homogeneous) so that it can be seen clearly images of organs recorded in the film.

Several previous studies that have examined X-Ray. Monitoring of radiation dose distribution using RPL in a glass dosimeter - Its application to radioactive emergency sensing was investigated by Nanto [1]. Dual energy CT dosimetry to detect acute-stage cerebral infarction: A phantom study was investigated by Hara [2]. Independent X-ray sensors for extreme environments were studied by Mohamed, Wright and Horsfall [3]. The design of a high dynamic range charge-todigital integrated converter for online dosimetry in radiotherapy was investigated by Gallin-Martel [4]. The dosimetric evaluation of proton CT using a prototype proton CT scanner was investigated by Giacometti [5]. The coded aperture design for super resolution compressed X-ray tomography was investigated by Mojica, Pertuz and Arguello [6]. A Deep Convolutional Approach to Low-Dose CT Image Noise Reduction was investigated by Badretale [7].

Monitoring Tumor Pulmonary Irradiation With Megavoltage Radiation Scattered Patients: A Complete System Simulation Study was investigated by Simoes [8]. XRay Acoustic Based Dosimetry Using Focused Ultrasound Transducers and Medical Linear Accelerators was investigated by Kim [9]. Efficient mammographic mass segmentation technique: a review researched by Kshema, George and Dhas [10]. Glucose-coated silver nanoparticles enter HeLa cells and cause S and G2 / M capture investigated by Panzarini [11]. Ion implantation in thermoplastic polymers was investigated by Di Benedett [12]. The measurement of continuous radiographic sources and intense flash with a Compton spectrometer was investigated by Gehring [13]. The Measurement of Voltage and Indirect Exposure Time for Medical X-rays was investigated by Bunkum, Pintavirooj and Visitsattapongse [14]. Chen [15] studied three-dimensional imaging of electric trees in various stages.

Comparison of Image Enhancement Method for Lumbar Spine X-ray Image was investigated by Saenpaen, Arwatchananukul and Aunsri [16]. A Bayesian approach to Anti-scatter Grid Extraction in X-ray Imaging was investigated by Cotte [17]. Reduction of X-Ray Dose in Chest Tomosynthesis was investigated by Miroshnychenko [18]. Generating a Synthetic X-Ray Image of a Person from Surface Geometry was investigated by Teixeira [19]. Classification of modalities and detection of concepts on medical images using distance transfer learning was investigated by Singh [20]. The Feasibility of X-ray Fluorescence Computed Tomography (XFCT) Imaging of Human Lung Tumors loaded with Gold Nanoparticles: The Monte Carlo Study was investigated by Ahmed, Jayarathna and Cho [21]. The Effect of Regularization Parameters in a Non-blind Restoration Algorithm Using Modified Iterative Wiener Filters for Medical Images was investigated by Sheer and Al-Ani [22]. Image Processing for X-ray Calibration Phantom was investigated by Wanluk, Pintavirooj and Treebupachatsakul [23]

The development of Multi-Axis X-ray CT for the Reduction of Metal Artifacts was investigated by Toru and Koseki [24]. Applying the Multi-CNN model to detect 
abnormal problems on chest X-rays was investigated by Kieu [25]. The Adaptive Generation of Structured Medical Reports Using NER Regarding Deep Learning was investigated by $\mathrm{Wu}$ [26]. Information entropy measurements and clustering improve edge detection in medical X-ray images studied by Hrzic [27]. Contrast Enhancement of Medical Radiographic Images Using Edge Preserving Filters was investigated by Kumari and Kanhirodan [28]. Simulated CT scanners for educational purposes were studied by Juhong and Pintavirooj [29].

This research aims to make an X-Ray film reader that can be used portable and equipped with a sensor of the presence of film and can be regulated by the digital light control. It also aims to provide convenience for operators / doctors to read the results of X-Ray films with light brightness that can be adjusted according to the needs of the operator / doctor.

\section{METHOD}

Block diagrams are made to map the process of a work. Block diagram functions to make it easier for someone to understand how it works itself. The block diagram of the XRay film reader is shown in Figure 1. From this figure, it can be seen that the Arduino microcontroller is used to read the $\mathrm{X}$ ray film sensor.

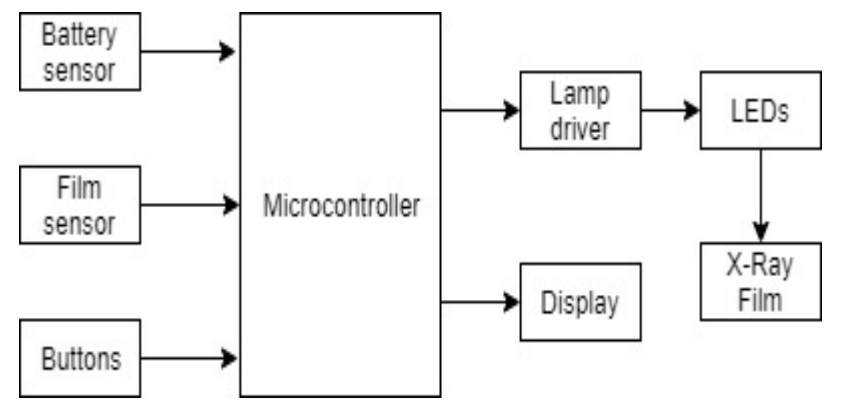

Fig. 1. System block diagram

The infrared transmitter emits waves that are fired at the When the On / Off button is turned on, the voltage from the 12 volt battery will enter the lamp driver circuit and the step down circuit, the voltage entering the step down circuit will be lowered and stabilized to 5 volts to supply the microcontroller. When the film is placed, the film sensor will detect and the LED light will turn on, then when there is no film the LED light will turn off automatically. The intensity of the light can be adjusted according to needs so that the film can be read clearly. There are choices of light intensity that are low, medium, and high..

\section{A. System work flow program}

A flowchart displayed on Figure 2 is explaining the system's work flow. When the device is turned on or the first power on is LCD initialization then the film reading sensor will work and detect X-Ray film, when the film is detected by the sensor the LED light will turn on and when the film sensor does not detect the film then the LED light will turn off. After that setting the light intensity, there are three choices of light intensity, namely low, medium, high. After the film reading is complete and the $\mathrm{x}$-ray film is removed from the device, the LED light will turn off automatically.

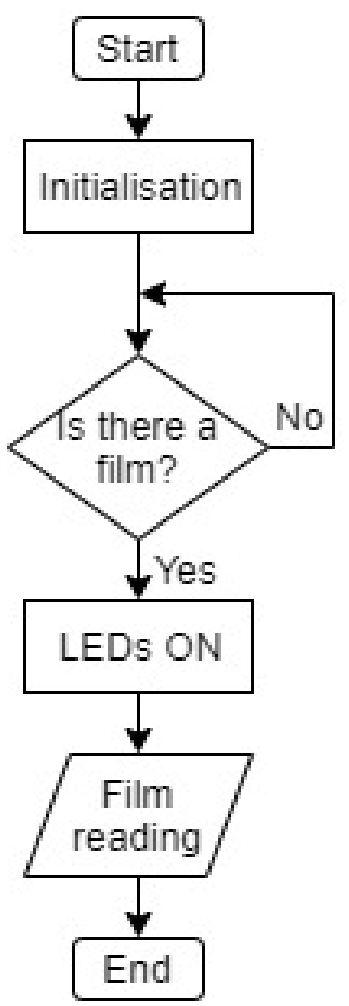

Fig. 2. System work flow diagram

\section{B. System hardware}

Making the schematic circuit functions to simulate the circuit to be able to ensure the circuit works according to what researchers expect. Making this series schematic using proteus application program, the application is used because proteus in its operation is easy to understand.

The minimum system circuit is a piece of hardware that functions as a series of targets to download or delete a program and as a tool executor, where there is an active component of IC ATMega8 as a place for the program to be planted.

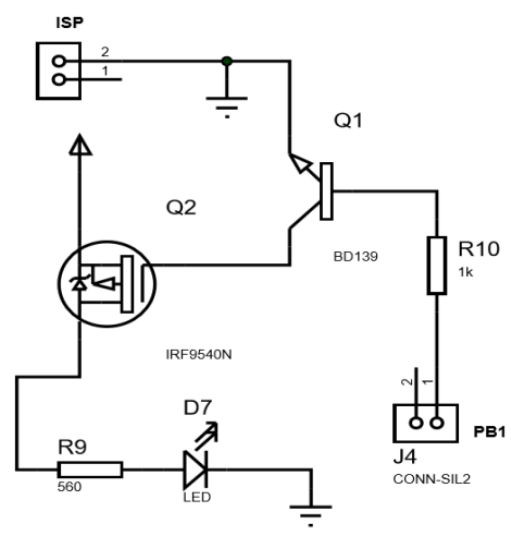

Fig. 3. Lamp driver schematic

The lamp driver circuit functions as power to turn on the LED light when getting high logic from the ATMega8 microcontroller IC shown in Figure 3.From this figure, it can be seen that the lamp driver uses transistor type BD139 and mosfet type IRF9540. 


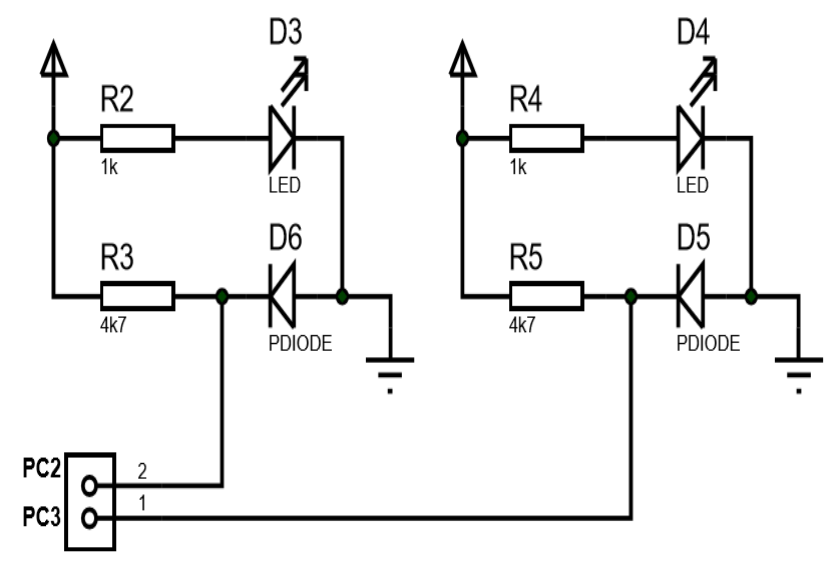

Fig. 4. Film sensor schematic

The film sensor circuit consists of a photodioda and infrared that serves to detect the film from the difference in the intensity of the light captured by the photodioda when there is resistance from the film and will turn on the LED lights is shown in Figure 4. From the figure, it can be seen that the photodiode circuit is connected in series using a 330ohm resistor.

\section{IMPLEMENTATION}

\section{A. Data analysis technique}

This testing tool aims to determine the limits of the ability of the tool in accordance with its function. The test data provided is to determine the quality and ability of the X-Ray viewer to read film.

This testing tool aims to determine the limits of the ability of the tool in accordance with its function. The test data provided is to determine the quality and ability of the X-Ray viewer to read film. The light intensity test on the module is carried out at 9 points 5 times in each mode shown in figure 5. From the picture it can be seen that test uses a lux meter in data collection, then tests the uniformity of the data which aims to test the uniformity of existing data. Then from the test results are sought averages, standard deviations, upper control limits, and lower control limits.

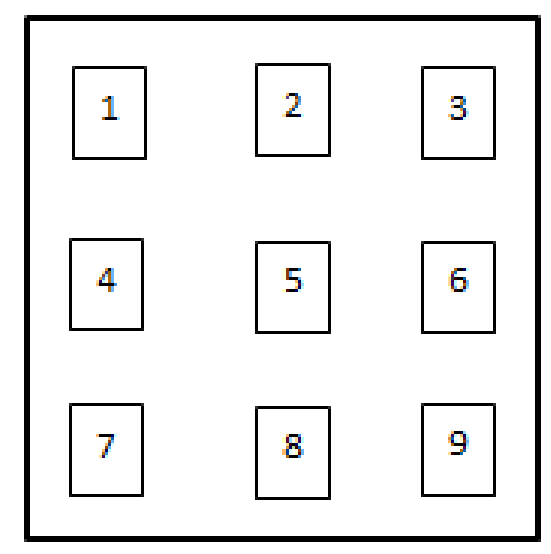

Fig. 5. Light intensity measurement points

The graph of the Uniformity of High Light Mode Intensity Data Measurement is shown in Figure 6. From the calculation results and from the picture it can be seen that the average of each subgroup is between the upper class limit and the lower class limit, so that the measurement of light intensity in high mode is uniform.

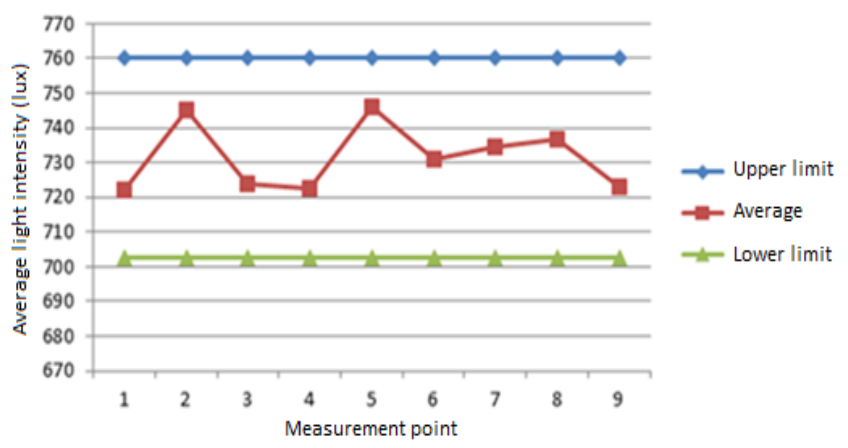

Fig. 6. Graph Uniformity Measurement Data Intensity of High Light Mode

The Graph of Moderate Light Mode Data Intensity Measurement Uniformity is shown in Figure 7. From the calculation results and from the picture it can be seen that the average of each subgroup is between the upper class limit and the lower class limit, so that the measurement of light intensity in high mode is uniform.

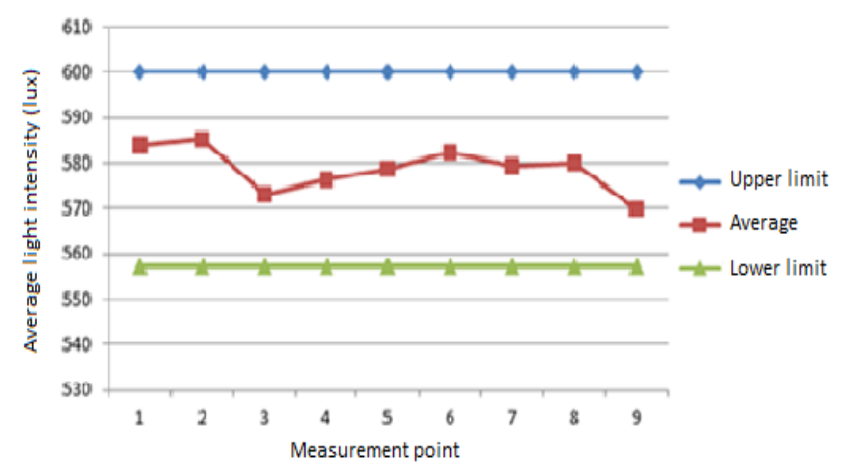

Fig. 7. Graph Uniformity Measurement Data Intensity of Medium Light Mode

The Graph of Low Light Mode Data Intensity Measurement Uniformity is shown in Figure 8. From From the calculation results and from the picture it can be seen that the average of each subgroup is between the upper class limit and the lower class limit, so that the measurement of light intensity in high mode is uniform.

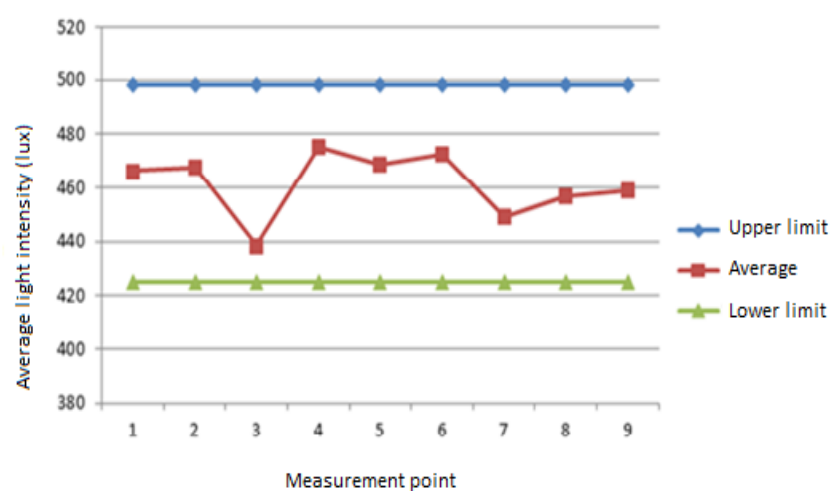

Fig. 8. Graph Uniformity Measurement Data Intensity of Low Light Mode

\section{Functional device performance test}

The X-Ray viewer created by the author is a tool used to assist a doctor or radiographer in reading X-Ray films. The research method was conducted by asking the opinions of 20 radiographers on the function and ability of the film viewer to 
read X-Ray films. Here are the test data that the writer can present based on test results from the radiographer.

The steps for operating the tool are as follows:

1) Turn on the appliance by pressing the ON / OFF button to the $\mathrm{ON}$ position

2) Put the X-ray film on the device, the light will turn on automatically

3) Adjust the light intensity on the settings button as needed

4) If the film reading has finished removing the X-ray film from the appliance, the lamp will turn off automatically

5) When the tool usage is complete, turn the device off by pressing the ON / OFF button to the OFF position.

Figure 9 is an X-ray film reader when the film is installed, the device is already on and the film reading lights turn on automatically because the sensor on the device detects the presence of the film.

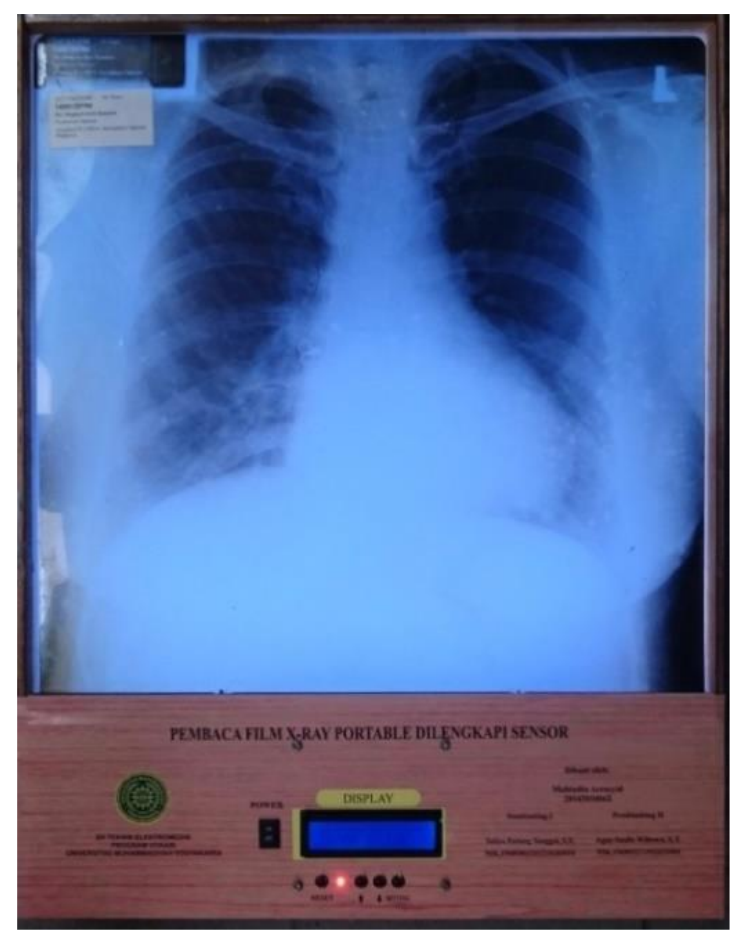

Fig. 9. Operational device visualization

\section{CONCLUSION}

Based on the analysis of data from 20 users, it can be concluded that the tools made by the author are easy to use, the results of the reading of the film are clear, and the tool is suitable for use in reading X-Ray films.

From the analysis results of the calculation of light intensity measurement data it can be concluded that the results of calculations and graphs show that the average of each light intensity mode is between the upper control limit and the lower control limit, so that the data obtained are uniform

In general it can be concluded that the tool that has been made by the author is in accordance with the initial planning, the tool can be used portable, the sensor can detect the presence of film and the light intensity can be set in 3 modes namely low, medium, and high.

\section{REFERENCES}

[1] H. Nanto et al., "Monitoring of radiation dose distribution utilizing RPL in glass dosimeter - Its application to radioactive emergency sensing-," in 2017 IEEE SENSORS, 2017, pp. 1-3.

[2] H. Hara et al., "Dosimetry of dual-energy CT for the detection of acutestage cerebral infarction: A phantom study," in 2016 IEEE Nuclear Science Symposium, Medical Imaging Conference and RoomTemperature Semiconductor Detector Workshop (NSS/MIC/RTSD), 2016, pp. 1-4.

[3] N. S. Mohamed, N. G. Wright, and A. B. Horsfall, "Self-powered XRay sensors for extreme environments," in 2017 IEEE SENSORS, 2017, pp. 1-3.

[4] L. Gallin-Martel et al., "Design of a high dynamic range integrated charge to digital converter for online dosimetry in radiotherapy," in 2016 IEEE Nuclear Science Symposium, Medical Imaging Conference and Room-Temperature Semiconductor Detector Workshop (NSS/MIC/RTSD), 2016, pp. 1-5.

[5] V. Giacometti et al., "Dosimetric evaluation of proton CT using a prototype proton CT scanner," in 2016 IEEE Nuclear Science Symposium, Medical Imaging Conference and Room-Temperature Semiconductor Detector Workshop (NSS/MIC/RTSD), 2016, vol. 7, pp. 1-3.

[6] E. Mojica, S. Pertuz, and H. Arguello, "Coded aperture design for super-resolution compressive X-ray tomography," in 2017 IEEE 7th International Workshop on Computational Advances in Multi-Sensor Adaptive Processing (CAMSAP), 2017, vol. 2017-Decem, pp. 1-5.

[7] S. Badretale, F. Shaker, P. Babyn, and J. Alirezaie, "Deep Convolutional Approach for Low-Dose CT Image Noise Reduction," in 2017 24th National and 2nd International Iranian Conference on Biomedical Engineering (ICBME), 2017, no. December, pp. 1-5.

[8] H. Simoes et al., "Monitoring Tumor Lung Irradiation With Megavoltage Patient-Scattered Radiation: A Full System Simulation Study," IEEE Trans. Radiat. Plasma Med. Sci., vol. 1, no. 5, pp. 452459, Sep. 2017.

[9] J. Kim et al., "X-Ray Acoustic-Based Dosimetry Using a Focused Ultrasound Transducer and a Medical Linear Accelerator," IEEE Trans. Radiat. Plasma Med. Sci., vol. 1, no. 6, pp. 534-540, Nov. 2017.

[10] Kshema, M. J. George, and D. A. S. Dhas, "Efficient mammographie mass segmentation techniques: A review," in 2017 International Conference on Wireless Communications, Signal Processing and Networking (WiSPNET), 2017, vol. 2018-Janua, pp. 2253-2256.

[11] E. Panzarini et al., "Glucose capped silver nanoparticles enter HeLa cells and induce S and G2/M arrest," in 2015 1st Workshop on Nanotechnology in Instrumentation and Measurement (NANOFIM), 2015, pp. 72-76.

[12] F. Di Benedett et al., "Ion Implantation in thermoplastic polymers," in 2015 1st Workshop on Nanotechnology in Instrumentation and Measurement (NANOFIM), 2015, pp. 238-241.

[13] A. Gehring et al., "Measurement of intense continuous and flash radiographic sources with Compton spectrometers," in 2017 IEEE 21st International Conference on Pulsed Power (PPC), 2017, vol. 2017June, pp. 1-3.

[14] M. Bunkum, C. Pintavirooj, and S. Visitsattapongse, "Indirect Measure Voltage and Exposure Time Device for Medical X-ray," in 2018 11th Biomedical Engineering International Conference (BMEiCON), 2018, pp. $1-4$.

[15] S. Chen, Z. Lv, S. M. Rowland, J. Carr, and P. J. Withers, "Three dimensional imaging of electrical trees in multiple stages," in 2017 IEEE Conference on Electrical Insulation and Dielectric Phenomenon (CEIDP), 2017, vol. 2017-Octob, pp. 425-428.

[16] J. Saenpaen, S. Arwatchananukul, and N. Aunsri, "A Comparison of Image Enhancement Methods for Lumbar Spine X-ray Image," in 2018 15th International Conference on Electrical Engineering/Electronics, Computer, Telecommunications and Information Technology (ECTICON), 2018, pp. 798-801.

[17] F. Cotte, M. Desvignes, H. Ayasso, and J.-M. Vignolle, "A Bayesian Approach for Anti-Scatter Grid Extraction in X-ray Imaging," in 2018 25th IEEE International Conference on Image Processing (ICIP), 2018, pp. 1183-1187.

[18] S. Miroshnychenko, O. Miroshnychenko, A. Nevgasymyi, and Y. Khobta, "Reduction of X-Ray Doses in Chest Tomosynthesis," in 2018 IEEE 38th International Conference on Electronics and Nanotechnology (ELNANO), 2018, no. 1, pp. 398-401. 
[19] B. Teixeira et al., "Generating Synthetic X-Ray Images of a Person from the Surface Geometry," in 2018 IEEE/CVF Conference on Computer Vision and Pattern Recognition, 2018, pp. 9059-9067.

[20] S. Singh, K. Ho-Shon, S. Karimi, and L. Hamey, "Modality Classification and Concept Detection in Medical Images Using Deep Transfer Learning," in 2018 International Conference on Image and Vision Computing New Zealand (IVCNZ), 2018, pp. 1-9.

[21] M. F. Ahmed, S. Jayarathna, and S. H. Cho, "Feasibility of X-ray Fluorescence Computed Tomography (XFCT) Imaging of Human Lung Tumors loaded with Gold Nanoparticles: A Monte Carlo Study," in 2018 IEEE 12th International Conference on Nano/Molecular Medicine and Engineering (NANOMED), 2018, pp. 250-254.

[22] A. H. Sheer and A. A. Al-Ani, "The Effect of Regularization Parameter within Non-blind Restoration Algorithm Using Modified Iterative Wiener Filter for Medical Image," in 2018 1st Annual International Conference on Information and Sciences (AiCIS), 2018, pp. 77-81.

[23] N. Wanluk, C. Pintavirooj, and T. Treebupachatsakul, "Image Processing for X-ray Calibration Phantom," in 2018 11th Biomedical Engineering International Conference (BMEiCON), 2018, no. 1, pp. 14.

[24] K. Toru and M. Koseki, "Development of a Multi-Axis X-Ray CT for Metal Artifact Reduction," in 2018 57th Annual Conference of the
Society of Instrument and Control Engineers of Japan (SICE), 2018, pp. 1344-1349.

[25] P. N. Kieu, H. S. Tran, T. H. Le, T. Le, and T. T. Nguyen, “Applying Multi-CNNs model for detecting abnormal problem on chest $\mathrm{X}$-ray images," in 2018 10th International Conference on Knowledge and Systems Engineering (KSE), 2018, pp. 300-305.

[26] C.-T. Wu, H.-K. Chang, J.-H. Liu, and J.-S. R. Jang, “Adaptive Generation of Structured Medical Report Using NER Regarding Deep Learning," in 2018 Conference on Technologies and Applications of Artificial Intelligence (TAAI), 2018, pp. 10-13.

[27] F. Hrzic, V. Jansky, D. Susanj, G. Gulan, I. Kozar, and D. Z. Jericevic, "Information entropy measures and clustering improve edge detection in medical X-ray images,” 201841 st Int. Conv. Inf. Commun. Technol. Electron. Microelectron. MIPRO 2018 - Proc., pp. 164-166, 2018.

[28] L. S. Kumari and R. Kanhirodan, "Contrast Enhancement of Medical Radiography Images Using Edge Preserving Filters,” in 2018 Fourth International Conference on Biosignals, Images and Instrumentation (ICBSII), 2018, no. March, pp. 206-212.

[29] A. Juhong and C. Pintavirooj, "Simulated CT scanner for educational purpose," in 2016 9th Biomedical Engineering International Conference (BMEiCON), 2016, pp. 1-4. 